\title{
NIVEL Y VARIACIÓN DE LA EQUIDAD EN LA EDUCACIÓN MEDIA DE ARGENTINA
}

\author{
Rubén Cervini \\ Universidad Nacional de Quilmes, Argentina
}

\section{INTRODUCCIÓN}

La igualdad de oportunidad educativa es un principio clave del pensamiento democrático y de la justicia solidaria en las sociedades capitalistas. Su aplicación a los 'resultados educativos' logros de aprendizaje), en contraposición a los 'insumos educativos', ha sido uno de los principales ejes de discusión académica sobre (in)equidad educativa. En este artículo se presentan los resultados del análisis de una extensa base de datos sobre los resultados obtenidos por los alumnos en una prueba estandarizada de Matemática del último año de la educación Media en Argentina. El estudio está centrado en la (in)equidad en los resultados educativos, concepto explorado a través de ciertos procedimientos 'correlacionales' propios de los modelos jerárquicos lineales.

Hace más de una década algunos organismos internacionales vienen abogando por un desarrollo con equidad en la región latinoamericana, particularmente referida a una 'mayor equidad en las oportunidades educativas' (CEPAL/UNESCO, 1992). En Argentina, el principal objetivo establecido en 1993 por Ley Federal de Educación ( $n^{\circ}$ 24.195) para la Educación General Básica, fue "Proporcionar una formación básica común a todos los niños y adolescentes del país garantizando su acceso, permanencia y promoción y la igualdad en la calidad y logros de los aprendizajes." (Art. 15, inc. a).

Según esta formulación, todos los individuos deberían tener asegurado el aprendizaje de las competencias básicas. Cualquier desigualdad en el logro, independientemente de su causa, debería considerarse como violación del principio de igualdad equitativa de posibilidades. Pero, a partir de esta igualación inicial básica, ideal, no toda desigualdad de aprendizaje violará los principios de la justicia distributiva, siempre y cuando sea consecuencia de ventajas proporcionadas por las dotes naturales (capacidad y talento) y la voluntad de emplearlas (aspiración y esfuerzo) (Rawls, 1971). Esta distribución desigual de las probabilidades en los resultados educativos del nivel post-primario debe ser independiente de cualquier otro criterio de discriminación (origen social, género, raza, ubicación geográfica). Tal premisa remite a los denominados "principios distributivos vinculantes", consistentes con el enfoque solidario de la justicia y preocupados por el requerimiento de interrumpir o suprimir la "relación entre dos variables" (Van Parijs,1992:213). Su forma 'negativa' reclama que el origen social del estudiante no afecte la distribución de los resultados educativos, razonamiento extensible a cualquier otro factor de inequidad (ej. género).

Los estudios dirigidos a determinar la magnitud de la correlación estadística entre los logros educativos del alumno y los factores individuales de inequidad (origen social, género, etnia, etc.) constituyen, de hecho, aproximaciones operacionales al problema central de la justicia distributiva en educación (grado en que se vola el "principio vinculante negativo"). Pero, al mismo tiempo, este tipo de estudios conlleva constataciones acerca del papel desempeñado por la escuela. Las investigaciones de 
Coleman et. all. (1966) y de Jencks (1972) son dos ejemplos clásicos a este respecto. La principal conclusión de ambos estudios fue que las diferencias en los resultados escolares se debían principalmente al origen social del estudiante, "todo lo demás - recursos financieros de la escuela, sus políticas, las características de los maestros - es o secundario o completamente irrelevante" (256). Con base en estos resultados, inicialmente se enfatizó la igualdad en los resultados como definición de la igualdad en la oportunidad educativa (IOE) y por tanto, "las diferencias de logros (entre estudiantes negros y blancos) son inequidades de oportunidad por definición" (Coleman,1969:21), es decir, en la situación ideal de la IOE "el medioambiente familiar de los blancos (clase media) y el de los negros (clase baja) no producirán efectos sobre el logro ..." (23). Pero, posteriormente, al reconocer la imposibilidad de alcanzar la igualdad en el resultado dado la fuerza del efecto de las diferencias familiares, reconceptualiza a la IOE como la reducción de "las desventajas que los niños enfrentan (en la escuela) en función de su primer medioambiente sin comprometer al sistema educativo con un fin inalcanzable" (Coleman, 1975:28). Se propone entonces, cambiar la condición de 'igualdad' por la de 'reducción de la desigualdad', y de esta forma, el concepto de IOE converge con el "principio distributivo vinculante" derivado de la teoría de la justicia distributiva, al tiempo que involucra a la escuela en razón de su mayor o menor eficacia redistributiva.

El reciente desarrollo de la técnica 'correlacional' de modelos jerárquicos lineales o 'multinivel' (Aitkin y Longford,1986; Bryk y Raudenbush,1992; Goldstein,1995) ha permitido operacionalizar otros aspectos del concepto de (in)equidad educativa, además de la simple correlación: (i) al modelar simultáneamente los diferentes niveles de agregación (por ejemplo: alumno, escuela), permite saber qué proporción de la variación del logro del alumno se debe principalmente a variables propias de cada nivel de agregación; (ii) a diferencia de los métodos tradicionales (OLS), es posible estimar sin sesgos el efecto de variables 'contextuales' (Goldstein,1995) de inequidad, que, adicionado al efecto de los factores individuales de inequidad, conforman la magnitud de (in)equidad total; (iii) permite saber si la magnitud del efecto de los factores de inequidad varía dentro de los diversos niveles de agregación (Lam, Wong y Ho Lai-ming, 2002; Nuttall, Goldstein, Prosser. y Rasbash, 1989; Opdenakker y Van Damme, 2001; Sammons, Thomas y Mortimore, 1997; Schreiber, 2002); (iv) dado que la variación en cada nivel está especificada en términos de los coeficientes de las variables explicativas (Goldstein,1998), permite determinar los efectos de los factores de inequidad sobre aquella variación (Lee y Bryk,1998; Schreiber,2002); (v) permite estimar de forma insesgada las posibles interacciones entre los factores individuales y 'contextuales' de inequidad Lee y Bryk, 1998; Opdenakker y Van Damme, 2001); (vi) al estimar los 'residuos' por niveles y para diferentes grupos de alumnos, es posible conocer la 'eficacia distributiva' de las escuelas (Lee y Bryk, 1998; Sammons, Thomas y Mortimore, 1997; Thomas, 2001).

En el presente trabajo se investiga la distribución del logro en Matemática de los alumnos del último año de la educación secundaria en Argentina, explorando todos los aspectos de la(in)equidadeducativa mencionados anteriormente, con excepción del último. Se utilizan los datos del Censo Nacional de Finalización del Nivel Secundario, realizado a finales del año lectivo de 1998 por el Ministerio de Cultura y Educación de la Nación. Con estos datos se pretende responder a los siguientes interrogantes específicos: (i) ¿Existen diferencias entre las escuelas respecto del rendimiento promedio en Matemática de los alumnos del último año de la educación media de Argentina?; (ii) ¿Cuál es la magnitud de la incidencia de los factores de inequidad del alumno individual - origen social, género y antecedente académico - sobre ese rendimiento?; (iii) ¿La intensidad de esa incidencia varía entre las escuelas?; (iv) ¿Cuál es la magnitud de la 
incidencia de la 'composición' institucional por factores de inequidad (origen social, género y antecedente académico) sobre el rendimiento promedio de las escuelas?; (v) ¿Existe interacción entre el efecto de los factores individuales de inequidad y los 'contextos'?; (vi) ¿La 'composición' de la escuela influye sobre la magnitud del efecto de los factores de inequidad del alumno individual?

\section{METODOLOGIA}

Datos. Provienen de (i) la prueba de Matemática y (ii) el Cuestionario del estudiante del Censo Nacional de Finalización del Nivel Secundario de 1998, realizado por el Ministerio de Cultura y Educación de la Nación. Los instrumentos fueron auto-aplicados. En el análisis se incluyen solamente estudiantes en escuelas de las modalidades bachillerato o comercial ${ }^{1}$, y con información válida para 20 o más estudiantes. Bajo estas condiciones, el archivo queda conformado por 126.590 estudiantes en 2.141 escuelas de 25 Estados $^{2}$.

Variables. La variable dependiente o 'criterio' es el puntaje obtenido por el alumno en la prueba estandarizada de Matemática. Las variables independientes son características del alumno individual y de 'composición' de la escuela:

(i) Las variables individuales del alumno se refieren a 'capital económico familiar', 'capital cultural familiar', sexo, antecedentes académicos y horas diarias de trabajo. Se definen de la siguiente forma:

○ (bienes+servicios): disponibilidad $(S i ́=1 ; \mathrm{No}=0)$ de 14 bienes de uso durable y servicios en el hogar;

- (educacion padres): suma del nivel educativo del padre y de la madre (14 puntos); $1=$ ninguno a 7 = universitario completo. Cuando la información del padre (madre) es 'missing', se asigna el valor de la madre (padre);

○ (libros+didácticos): compuesto por

- (libros): disponibilidad de libros en el hogar: $1=$ menos de $10 ; \ldots ; 5$ =más de 100;

- (didacticos): disponibilidad de libros, fichas y apuntes escolares: 1=ninguno; 2= algunos; 3=todos;

- Procedimiento de conformación de (libros+didácticos): (i) se recodifica (libros). $1=0 ; 2=0.20 ; \ldots$ ; $5=0.80$ y (ii) se adiciona a (didácticos).

○ (femenino): mujeres $=1$; hombres $=0$;

○ (repitiente): $1=$ alumno que repitió al menos una vez; $0=$ alumno que no repitió;

○ (hs_trabajo): cantidad de horas por día que el estudiante dedica a trabajar; 0 = ninguna; 1 = hasta 2 hs.; ...; 5 = 5 o más hs.

\footnotetext{
${ }^{1}$ Las escuelas técnicas no son incluidas en el análisis porque tienen importantes diferencias curriculares y de resultados con respecto a las otras modalidades (ver Cervini, 2003b).

${ }^{2}$ El estado de Buenos Aires se divide en Gran Buenos Aires (Conurbano) y resto del Estado.
} 
(ii) Las variables de 'composición escolar' son los promedios escolares de cada variable individual del estudiante, excepto el género y la repitencia ('dicotómicas'), para las cuales se usa el porcentaje de la escuela.

Breves comentarios sobre las variables. Los bienes y servicios en el hogar ('capital económico') y el nivel educativo alcanzado por los padres ('capital económico y cultural') han sido extensamente utilizados como mediciones del origen social del alumno y en general, registran una alta asociación con el rendimiento escolar. Este es también el caso de 'libros en el hogar' ('capital cultural objetivado') y de 'libros y materiales didácticos escolares'. Sin embargo, se decidió utilizar una combinación sumativa de ambos porque la misma poseía una capacidad predictiva superior a la obtenida cuando ambos indicadores actuaban separadamente.

Las diferencias de género en el logro de matemática es un tema recurrentemente investigado. En su revisión de un centenar de investigaciones, Friedman (1989) concluye que no hay diferencia entre sexos, y si la hay, es a favor de las mujeres. Para los primeros años de la secundaria, algunas investigaciones informan ventajas para las mujeres (Tsai y Walberg,1983), otras para los hombres (Hilton y Berglund,1974) y otras para ninguno (Fennema y Cerpenter,1981). Para el final del secundario, sin embargo, la gran mayoría de las investigaciones reporta ventajas para los hombres (Friedman,1989).

Dado que no se dispone de ningún indicador específico del nivel de logro académico antecedente del alumno, se utiliza el episodio de repitencia como un indicador proxy.

Más allá de algunas inconsistencias (Quirk, Keith y Quirk, 2002), la investigación ha coincidido en que el efecto del trabajo extra-escolar sobre el desempeño académico depende de las horas de trabajo. En general, se detectan efectos negativos cuando la dedicación al trabajo supera 15-20 horas semanales (Cooper, Valentine, Nye y Lindsay,1999; Kablaoui y Pautler,1991; Marsh, 1991; Steinberg y Kaufman,1995; Steinberg y Dornbush, 1991). Algunos estudios longitudinales han sido también convergentes con esa conclusión (Mortimer, Finch, Ryu, Shanahan y Call,1996; Quirk, Keith y Quirk,2002; Singh,1998). La interpretación más plausible es que a medida que aumentan las horas de trabajo, disminuye la "oportunidad de aprender" (Carroll,1963), uno de los principales condicionantes del nivel de rendimiento ${ }^{3}$. Este razonamiento, sin embargo, debería relativizarse. Una revisión de investigaciones mostró que los alumnos involucrados en actividades extra-curriculares (como deportes) tienen logros más altos (Holland y Andre,1987), conclusión confirmada por investigaciones más recientes (March,1992; Gerber,1996).

Técnica y estrategia de análisis. Para el análisis de las relaciones entre el rendimiento y las diferentes variables, se utiliza el programa MLwiN (Goldstein et.alli.,1998), basado en el método de "análisis estadístico por niveles múltiples" o "modelos jerárquicos lineales" (Aitkin y Longford, 1986; Bryk y Raudenbush, 1992; Goldstein,1995). Los datos permiten definir modelos con tres niveles de agrupamiento: el estudiante (nivel 1), la escuela (nivel 2) y el Estado (nivel 3). Este último nivel se incluye con el objeto de no sobreestimar la variación "entre-escuela", foco de interés de este trabajo. Los modelos explorados y su secuencia responden a las preguntas de investigación propuestas como objetivos específicos y a la técnica de análisis utilizada. La

\footnotetext{
${ }^{3}$ Para el caso argentino, ver Cervini (2001).
} 
determinación de la probabilidad del efecto de las variables se basa en el test de la razón de máxima verosimilitud ${ }^{4}$.

\section{RESULTADOS}

En el Cuadro 1 se presentan las estadísticas descriptivas de las variables individuales y agregadas, y en el Anexo A aparecen los coeficientes de correlación. Entre las variables individuales, las correlaciones más estrechas son las de educación de los padres con bienes en el hogar (0.507) y con disponibilidad de libros y didácticos (0.266). Las correlaciones entre 'horas de trabajo' y los otros indicadores de estatus socioeconómico y cultural del alumno son bajas, sugiriendo que aquel indicador expresa características o aspectos diferentes a los captados por los indicadores más tradicionales. Las correlaciones de las variables de 'contexto' escolar en general son elevadas, con excepción del porcentaje de alumnas en la escuela, la cual no se correlaciona con ninguna de las restantes.

\begin{tabular}{|c|c|c|c|c|}
\hline \multicolumn{5}{|c|}{$\begin{array}{l}\text { 1. - Estadísticas descriptivas de las variables individuales y } \\
\text { 'composicionales' }\end{array}$} \\
\hline Variables & Media & $\begin{array}{l}\text { Desvío } \\
\text { estándar }\end{array}$ & $\begin{array}{c}\text { Valor } \\
\text { mínimo }\end{array}$ & $\begin{array}{l}\text { Valor } \\
\text { máximo }\end{array}$ \\
\hline \multicolumn{5}{|l|}{ ALUMNO } \\
\hline femenino & 0.621 & 0.485 & 0 & 1 \\
\hline repitiente & 0.244 & 0.430 & 0 & 1 \\
\hline educación padres & 7.866 & 3.011 & 1 & 13 \\
\hline bienes+servicios & 8.359 & 2.573 & 1 & 14 \\
\hline libros+didacticos & 2.739 & 0.680 & 1 & 3,8 \\
\hline hs_trabajo & 0.960 & 1.463 & 0 & 4 \\
\hline \multicolumn{5}{|l|}{ ESCUELA* } \\
\hline femenino & 0.619 & 0.189 & 0 & 1 \\
\hline repitiente & 0.240 & 0.201 & 0 & 1 \\
\hline educación & 7.835 & 1.901 & 3,64 & 12,71 \\
\hline bienes+servicios & 8.388 & 1.556 & 3,17 & 13,23 \\
\hline libros+didacticos & 2.747 & 0.271 & 1,84 & 3,59 \\
\hline hs_trabajo & 0.960 & 0.573 & 0 & 3,55 \\
\hline
\end{tabular}

A seguir, la presentación de los resultados se organiza de acuerdo a los objetivos propuestos y a la estrategia de análisis adoptada. En cada punto se explica brevemente el procedimiento realizado. El Cuadro 2 contiene las estimaciones de todos los modelos, enumerados de acuerdo al objetivo específico correspondiente. En el Anexo B se encuentran las estimaciones con su error estándar correspondiente.

1. ¿Existen diferencias entre las escuelas respecto del rendimiento promedio en Matemática de los alumnos del último año de la educación media de Argentina? Para responder esta pregunta se estiman la variación alrededor de la media global del rendimiento (Parte fija) y simultáneamente, las variaciones en cada nivel de agregación (Estado, escuela y alumno) (Parte aleatoria). Esta partición inicial

\footnotetext{
${ }^{4}$ El grado de ajuste (probabilidad) de un modelo se estima en base a la diferencia entre los valores de la razón de máxima verosimilitud del modelo analizado y del modelo antecedente, diferencia que puede ser referida a la distribución de chi-cuadrado y cuyos grados de libertad quedan definidos por la cantidad de nuevos parámetros que han sido ajustados en el modelo analizado.
} 
de la varianza del rendimiento en 3 niveles no contiene ningún predictor. Los resultados en la Cuadro 2 indican que la variación estimada del rendimiento promedio de las escuelas $(=126,405)$ representa el $31,7 \%$ de la variación total del rendimiento (correlación intra-clase=97,521/39,437+97,521+218,446), estimación significativa $(p<.001)$ de acuerdo al test de máxima verosimilitud $(=1047903,0)$. La media global estimada es $52,05 \%$ (e.s. $=1,482$ ). Estos resultados no son novedosos puesto que ya habían sido informados en estudios anteriores (Cervini, 2002; 2003a). Entonces, existen diferencias significativas entre las escuelas respecto del rendimiento promedio de sus alumnos.

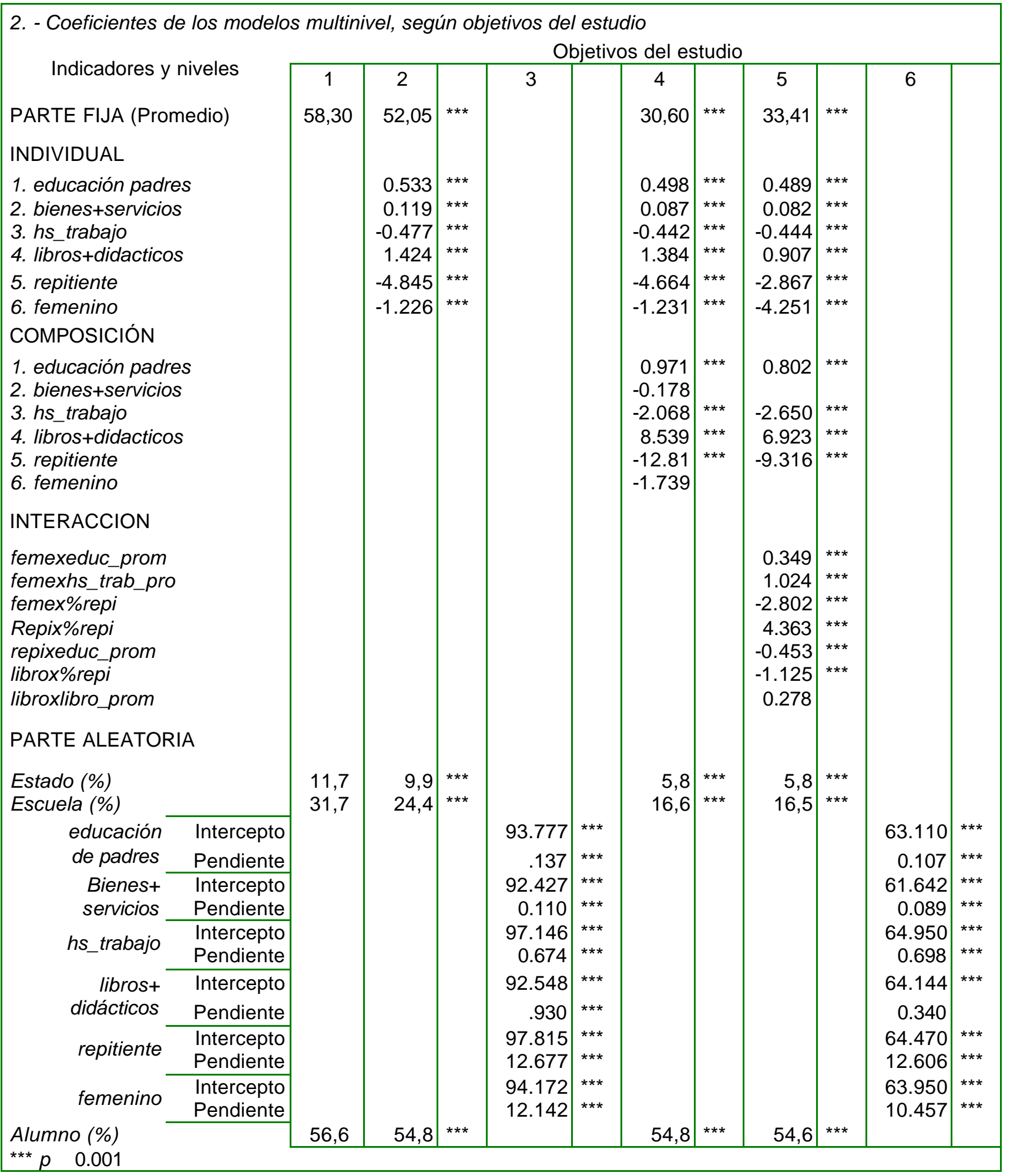


2. ¿Cuál es la incidencia de los factores de inequidad del alumno individual - origen social, género y antecedente académico - sobre ese rendimiento? Todas las variables individuales se incluyen simultáneamente en el modelo "vacío". Cada estimación indica el efecto promedio de la variable sobre el rendimiento, después de haber controlado a las restantes. En las variables 'dummy', el efecto es la diferencia esperada entre la categoría 'base' (no repitiente; hombre) y la variable 'dummy' (repitiente; femenino). Los resultados confirman estudios anteriores que indicaban una estrecha correlación de estos factores con el rendimiento (Cervini, 2002; 2003a). Las mujeres obtienen, en promedio, rendimientos menores al de los varones $(-1,23)$, situación similar al de los repitientes respecto de los no-repitientes $(=-$ $4,85)^{5}$. Las tres mediciones referidas al origen sociocultural de alumno mantienen un efecto positivo propio; por tanto, cuanto más alto sean el nivel educativo de los padres y/o el acceso del alumno a bienes de uso durable y servicios ('capital económico'), y/o la disponibilidad de bienes culturales y educativos, más alto será el rendimiento en Matemática. Finalmente, la estimación del efecto de las horas de trabajo y su signo negativo $(-0,477)$ indican que cuantas más horas trabaja el alumno, menor será su nivel de rendimiento. La estimación del efecto de cada una de estas variables es significativamente alta ( $p<.001)$. Este conjunto de variables individuales ha 'explicado' apenas el 3,5\% de la variación "entre-alumno" dentro de la escuela (226,032 - 218,45 / 226,032), y el 29,6\% de la variación "entre-escuela". O sea, su efecto mayor se detecta a nivel de las diferencias entre las escuelas, reflejando el alto grado de segmentación o selectividad institucional por características socioculturales de composición ${ }^{6}$.

3. ¿La fuerza de la incidencia de esos factores varía entre las escuelas? El modelo anterior suponía que la intensidad de la asociación entre rendimiento y cada uno de los factores individuales (pendiente â), era similar en todas las escuelas. Sin embargo, ella puede variar. Para evaluar esta posibilidad se debe permitir que tal correlación varíe (aleatorización) a nivel escuela. Dado que se pretende conocer no sólo la existencia de esta variación, sino también su posible relación con la 'composición' de la escuela (ver más adelante, Modelo 6), cada variable es 'aleatorizada' individualmente, es decir, se especifica un modelo para cada aleatorización. Las estimaciones del intercepto (promedio) y del efecto en la parte aleatoria son las varianzas de los promedios escolares alrededor de la media global del rendimiento y del efecto de la variable en cada escuela alrededor del efecto promedio estimado, respectivamente. Con el fin de simplificar el análisis, se supone la inexistencia de covariación entre intercepto y pendiente.

Todas las estimaciones de la varianza de los 'efectos' resultaron significativas. El efecto de cada variable sobre el rendimiento difiere entre las escuelas. Es decir, existen diferencias en el grado de (in)equidad institucional, reflejando disparidades en la capacidad institucional para compensar el efecto del origen social, el género o los antecedentes académicos del alumno. En algunas escuelas la pendiente de los indicadores del origen social es más inclinada que en otras. De la misma forma, la diferencia promedio de rendimiento entre géneros varía significativamente entre las escuelas $(=12,14)$, al igual que la diferencia promedio entre alumnos repitientes y no-repitientes $(=12,68)^{7}$.

\footnotetext{
${ }^{5}$ En las estimaciones de modelos individuales iniciales, sin "controles", la distancia no_repitientes / repitientes es -5,39, con un rendimiento promedio de $54,28 \%$ para los repitientes; mientras que la distancia entre géneros es de $-0,743$, con un rendimiento promedio de $58,02 \%$ para las mujeres.

${ }^{6}$ En general, se espera que las variables afecten principalmente a la varianza del nivel en el que están definidas. Así por ejemplo, las variables individuales del alumno deberían afectar principalmente a la varianza del nivel 'alumno'. Sin embargo, cuando la 'composición' de los grupos (escuela) respecto de las variables explicativas individuales no es igual para todos ellos, se producirá también una caída de la varianza a nivel de esos grupos ('inter-escuela'). Entonces, las variables explicativas del nivel individual (alumno) explicarán parte de la varianza individual y parte de la grupal.

${ }^{7}$ Esto significa que la dispersión de la diferencia hombre/mujer en torno de la media global $(-1,24)$ es 3,48, mientras que la diferencia no-repitiente/repitiente varía 3,56 en torno de la media global $(-4,82)$.
} 
El rendimiento promedio de los diferentes grupos de alumnos también varía significativamente. La variación del rendimiento promedio de las mujeres es 94,17, y tal cual se desprende de la variación de la distancia apuntada arriba, la variación del rendimiento promedio de los hombres es 106,31. De la misma forma, la variación del promedio de los repitientes $(97,82)$ es significativamente menor a la de los norepitientes $(110,492)$.

Para las variables restantes, asumidas como continuas, interesa saber cuál es el comportamiento de la varianza de la suma de los dos componentes estudiados (intercepto y pendiente) para cada uno de los valores de tales variables. A modo de ilustración, los Gráficos 1 y 2 presentan la varianza "entre-escuela" para cada valor de las variables educación de padres y libros+didácticos, respectivamente. Se observa que a medida que cada variable aumenta de valor, también lo hace la varianza respectiva. Es decir, existe una mayor variación entre las escuelas para los estudiantes con mayor estatus sociocultural. Las escuelas son más homogéneas para los alumnos de menos recursos, mientras que existe una mayor diferenciación en la efectividad escolar cuanto mayor es el estatus sociocultural de los alumnos ${ }^{8}$. Entonces, el rendimiento esperado de los alumnos con bajo 'capital cultural' (educación padres o libros+didacticos) será muy similar, no importa a qué escuela asista.

4. ¿Cuál es la magnitud de la incidencia de la 'composición' institucional de factores de inequidad (origen social, género y antecedente académico) sobre el rendimiento promedio de las escuelas? En el presente estudio, la 'composición' es una estadística resumen (la media o la proporción) de un agregado (escuela), relativa a una determinada variable (ej. educación de los padres) de las unidades individuales (alumnos) que conforman aquel agregado (escuela). Se entiende por efecto 'composición' la incidencia de esa estadística resumen sobre el rendimiento del alumno, siempre que previamente se haya considerado el efecto de la variable individual a la que se refiere (Goldstein,1995; Nutall et.alli.,1989). Puede interpretarse como el efecto que tiene la mayor (o menor) concentración de alumnos con determinada característica individual (por ejemplo, repitencia escolar) sobre el logro académico, adicional a las desigualdades esperadas en el logro dentro de la escuela atribuibles a las diferencias entre los alumnos respecto de aquella característica individual. Para responder a la pregunta formulada, todas las variables individuales 'agregadas' (porcentajes o promedios a nivel de escuela) se introducen simultáneamente en el Modelo 2, manteniendo el 'control' de los factores individuales del alumno. Los resultados se presentan en el Modelo 4.

La composición por género de la escuela no ayuda a predecir el rendimiento promedio de los alumnos. El rendimiento de una alumna cualquiera de una escuela no subirá (o bajará) si se sitúa en otra escuela, similar en todas las características consideradas, pero con diferente proporción de mujeres (o de hombres). Tampoco influye el contexto 'económico', medido a través del acceso a servicios y bienes de uso durable. Pero, en los aspectos restantes sí existe efecto contextual. De dos alumnos repitientes, se deberá esperar peor rendimiento de aquel que se encuentre en una escuela con mayor proporción de repitientes. De la misma forma, de dos jóvenes que trabajan igual cantidad de horas, deberá esperarse mejor rendimiento de aquel que se encuentre en una escuela con el menor promedio de horas de trabajo. El mismo tipo de conclusión es extensible a los indicadores de contexto cultural (educación padres y libros+didacticos).

\footnotetext{
${ }^{8}$ Esta conclusión está acotada a la variación del rendimiento promedio (intercepto) y del efecto del factor (pendiente), pero podría cambiar el sentido si se modelase también la covarianza de ambas variables.
} 
El conjunto de estos indicadores ha disminuido en 41,7\% la varianza 'inter-Estadual' (39,44$22,98 / 39,44)$ y en $32,1 \%$ la varianza 'entre-escuela' $(97,52-66,26 / 97,521)$ del Modelo 2. La variación "entrealumno" tal cual era esperado, no se ha modificado.

5. ¿Existe interacción entre el efecto de los factores individuales de inequidady el 'contexto' escolar? El procedimiento seguido es el siguiente: (a) todas las interacciones posibles se incluyen, una por vez, en el Modelo 4 ('composición'); (b) se seleccionan solamente las que resultan significativas y se las incluye simultáneamente. Los resultados aparecen en el Modelo 5. La constatación general más relevante es que todas las interacciones significativas involucran el género o la repitencia y por tanto, no existen interacciones entre los indicadores del origen sociocultural ${ }^{9}$. Las estimaciones y sus signos permiten extraer algunas conclusiones más específicas:

(a) A medida que aumenta el nivel educativo promedio de los padres o por el contrario, disminuye el promedio de horas de trabajo de los alumnos, menor es la diferencia entre géneros. Por otra parte, a medida que aumenta la proporción de repitientes, aumenta la distancia entre los géneros. De dos alumnas, aquella que se encuentre en un contexto sociocultural o académico más alto, tendrá mayor probabilidad de obtener un rendimiento más próximo al rendimiento promedio de los varones pertenecientes a ese mismo contexto. Entonces, la ventaja masculina en el rendimiento de Matemática se hace más pronunciado en los contextos sociales y académicos más bajos;

(b) El término interactivo repiXeduc_prom es significativo y por tanto, a medida que aumenta el nivel educativo promedio de los padres, aumenta la diferencia entre repitientes y no-repitientes. Algunos procesamientos iniciales sin "control" estadístico, demostraron que esta diferencia también varía y con el mismo sentido, a medida que varían los niveles promedio de la disponibilidad de libros y didácticos (repiXlibd\%) y de las horas que trabajan los alumnos (repiXtrab\%). Pero estas asociaciones se desvanecen cuando todas las interacciones se analizan conjuntamente. Por lo tanto, la estimación de repiXeduc_prom expresa fuertes y consistentes indicios de que el efecto de los antecedentes académicos de los alumnos es muy sensible al contexto sociocultural, aumentando a medida que éste es más ventajoso;

(c) A medida que disminuye el porcentaje de repitientes, la diferencia entre repitientes y norepitientes aumenta. Si bien podría presuponerse una superposición con la interacción anterior, el "control" estadístico confirma el efecto propio de repiX\%repi. Respecto de esta constatación, análisis realizados y no presentados aquí mostraron que (i) los alumnos repitientes obtienen más altos rendimientos a medida que el contexto académico mejora, pero (ii) el aumento relativo de esos rendimientos es menor que el observado en los alumnos no-repitientes. Por lo tanto, el efecto de los antecedentes académicos del alumno individual es sensible a la composición académica de la escuela, aumentando en contextos donde predominan alumnos sin fracasos escolares precedentes;

(d) Finalmente, se constata que cuando aumenta la proporción de repitientes, el efecto de los libros y materiales escolares disminuye. Este es un resultado sorprendente. Con el objeto de ilustrarlo, se calcula la varianza residual en el nivel alumno del siguiente modelo simplificado (Parte Fija), con aleatorización de la covarianza 'libros / \%repitiente' en el nivel 1 (alumno):

\footnotetext{
${ }_{9}^{9}$ La interacción libroXlibro_prom resultó significativa cuando actuaba aisladamente, pero dejó de serlo al incorporar (libroX\%repi).
} 


$$
\begin{aligned}
& \operatorname{matema}_{i j k} \sim \mathrm{N}(X B, \Omega) \\
& \text { matema }_{i j k}=\not \partial{ }_{0 i j} \text { cons }+\not{ }_{1 i} \text { libro+didactico } \\
& i j k
\end{aligned}
$$

, donde matema $_{\mathrm{ijk}}$ es el rendimiento en matemática del alumno $i$ en el escuela $j$ del Estado $k ; B_{1}, B_{2} y$ $B_{3}$ son parámetros (pendiente) asociados a libro+didáctico, \%repitientes y librox\%repite, respectivamente, a ser estimados; cons es una constante $=1$ y $\beta_{0 \mathrm{ijk}}$ es un parámetro asociado a cons, compuesto así: $\beta_{0 \mathrm{jik}}=$ $\beta_{0}+v_{0 k}+\mu_{0 j k}+e_{0 i j k}$, donde $\beta_{0}$ es el rendimiento promedio estimado (Parte fija), y $v_{0 k}, \mu_{0 j k}$ y $\epsilon_{0 i j k}$ son "residuos" a nivel alumno, escuela y Estado, respectivamente; o sea, cantidades aleatorias, no correlacionadas, normalmente distribuidas, con media $=0$ y cuyas varianzas respectivas $\left(\sigma_{v}, \sigma_{\mu}\right.$ y $\left.\sigma_{e}\right)$ han de estimarse. Dado que se está modelando la covarianza 'libros / \%repitiente' en el nivel alumno, la varianza del nivel alumno es la varianza de la suma de la varianza asociada a cons y de la covarianza libro*\%repitiente, es decir, $\sigma_{\mathrm{e}}=\sigma_{\mathrm{e} 0}$ cons $^{2}+2 \sigma_{\mathrm{e} 12}$ libro $_{\mathrm{ijk}} * \%$ repitiente $\mathrm{j}_{\mathrm{k}}$. El Gráfico 3 expone los resultados de este modelo. Se observa el aumento creciente positivo de la varianza con respecto al porcentaje de repitientes. Por lo tanto, la tenencia de libros y materiales escolares es un predictor más efectivo entre los alumnos de contextos con niveles académicos más elevados, y menos efectivo en los contextos académicos inversos.

6. ¿La 'Composición' de la escuela influye sobre la magnitud (variación) del efecto de los factores de inequidad individuales en el nivel escuela? Se trata de determinar si la variación del intercepto y de la pendiente de cada factor en el nivel escuela, detectada por el Modelo 3 , se asocia con la 'composición' de la escuela. Los resultados se presentan en el Modelo 6.

Consistente con resultados informados en trabajos anteriores (Cervini, 2002; 2003b), la variación del rendimiento promedio de la escuela (intercepto) cae abruptamente con todos los indicadores de composición sociocultural. Cada uno de ellos explica más del $30 \%$ de las diferencias de rendimiento entre las escuelas. El efecto sobre el nivel de (in)equidad institucional, en cambio, es más variado. La 'composición' de la escuela produce una disminución aproximada del $20 \%$ en la variación del efecto (pendiente) de los dos indicadores clásicos - bienes+servicios y educación padres. Pero, la variación de la fuerza predictiva de libros+didacticos sorprendentemente cae más del $60 \%$ (de 0.930 a 0.340 ), tornándose no significativa. Finalmente, la 'composición' consigue explicar un poco más del 10\% de la variación en la distancia entre géneros, y no muestra asociación con el efecto de la repitencia ni con las horas dedicadas al trabajo extra-escolar.

Entonces, los efectos 'intra-escuela' de los factores individuales de inequidad sociocultural serán más similares entre escuelas con la misma 'composición' que en la totalidad de las escuelas. Más aún, los rendimientos de los alumnos según la disponibilidad de libros y materiales escolares, serán iguales. Los rendimientos predichos por (educación padres) o por (bienes y servicios) se tornan también más homogéneos, pero en grados muy inferiores. Finalmente, los factores de 'composición' no guardan ninguna o muy poca relación con la variación de la eficacia institucional referida a la historia académica, al género o al tiempo diario de trabajo extra-escolar.

Los Gráficos 4 y 5 muestran las estimaciones de la varianza del Modelo 5, aleatorizando los efectos de educación padres y libros+didácticos, respectivamente. Al compararlos con los Gráficos 1 y 2 , 
respectivamente, se constata que efectivamente, los rangos de la varianza de ambas variables han disminuido, pero la caída del correspondiente a libros+didácticos es notablemente más acentuada.

\section{DISCUSIÓN Y CONCLUSIONES}

El análisis realizado en este trabajo estuvo orientado a profundizar el conocimiento de la distribución del logro escolar en matemática de acuerdo a ciertos factores de inequidad, como el origen social, el género y los antecedentes académicos. Por tanto, el estudio se preocupa por el grado en que se viola el principio democrático de la igualdad de oportunidades educativas.

Algunos hallazgos no son novedosos puesto que ya habían sido informados en trabajos anteriores. Los datos indicaron que el rendimiento promedio varía significativamente entre las escuelas. Es decir, el logro esperado de cada alumno dependerá en gran medida, de la escuela a la que asiste. Una alta proporción de esta variación se explica por el origen social, el género y los antecedentes académicos (repitencia) del alumno. Cuanto más alto sean el nivel educativo de los padres y/o el acceso del alumno a bienes de uso durable y servicios, y/o la disponibilidad de bienes culturales y educativos, más alto será el rendimiento en Matemática. A la inversa, cuantas más horas dedica el alumno al trabajo extra-escolar, menor será su nivel de rendimiento. Además, las mujeres y los repitientes obtienen, en promedio, rendimientos menores al de los varones y no repitientes, respectivamente. El efecto de estos indicadores de inequidad educativa, a pesar de estar definidos en el nivel 'alumno', se detecta principalmente por el descenso de las diferencias entre las escuelas que produce, comportamiento que refleja el alto grado de segmentación o selectividad institucional en el sistema educativo.

Se estableció también que, adicional a efecto de los factores individuales de inequidad, existen 'efectos contextuales', con excepción de la variable género (relación hombre/mujer en la escuela). Así por ejemplo, de dos alumnos repitientes similares, se deberá esperar el peor rendimiento de aquel que se encuentre en una escuela con mayor proporción de repitientes. Esta conclusión es válida también para los contextos definidos por las horas de trabajo, la educación de los padres y la disponibilidad de bienes culturales y educativos. Por eso, la variación 'inter-escuela' del rendimiento promedio cae abruptamente con todos los indicadores de composición sociocultural. Si bien es razonable aceptar que el origen de este efecto 'composición' se encuentra en factores extra-escolares, o sea, en características y procesos intrafamiliares externos a la propia escuela, no es menos cierto que la organización y las prácticas escolares internas tienden reproducir esos esquemas de determinación extra-escolar. En un análisis anterior de estos mismo datos (Cervini,2004) se ha demostrado que, a nivel del conjunto total de escuelas, el efecto 'composición' se superpone a las relaciones entre nivel de logro escolar y características y prácticas institucionales o pedagógicas de la escuela, siendo éstas simplemente mediadoras del efecto 'composición'.

El presente trabajo ha aportado nuevas evidencias sobre la diversidad en los patrones de distribución (in)equitativa del logro escolar. En primer lugar, la intensidad de (in)equidad institucional varía significativamente entre las escuelas. Éstas difieren en su capacidad para compensar el efecto del origen social, del género o de los antecedentes académicos del alumno. La distancia entre el rendimiento promedio de los hombre y de las mujeres, de los repitientes y de los no repitientes, de los trabajadores y no trabajadores, varía entre las escuelas. Como también lo hace la magnitud del efecto de todos los 
indicadores sobre el origen social del alumno. Puede concluirse entonces, que existen escuelas más 'igualadoras' que otras (Bryck y Raudenbush,1992)

En segundo lugar, existen fuertes indicios de que esta variación 'inter-escuela' se asocia con los factores de inequidad. La capacidad predictiva de los factores de inequidad por escuela varía según cual sea el nivel de 'capital cultural' del alumno, su género o historia de repitencia. En general, existe una menor variación entre las escuelas para los alumnos estudiantes con menor estatus sociocultural, mientras que a medida que éste aumenta, también lo hace la varianza no-explicada. Entonces, los rendimientos obtenidos por los alumnos con origen social menos favorables ('capital cultural' bajo) serán más próximo al promedio esperado para ese tipo de alumno en la escuela, que el de los alumnos de origen social más favorable respecto de su promedio esperado.

En tercer lugar, y consistente con el punto anterior, se verificó que en contexto más favorecidos social o académicamente, o donde los alumnos trabajan menos horas, la diferencia entre géneros se estrecha, es decir, la desventajas femeninas se acentúan en los contextos menos favorables.

En cuarto lugar, el promedio esperado de los alumnos repitientes aumenta a medida que el porcentaje de repitientes disminuye. Sin embargo, su aumento relativo es inferior al experimentado por los alumnos no-repitientes, por lo cual la distancia de rendimiento debida a los antecedentes académicos del alumno se acentúa a medida que los contextos sociocultural y/o académico se tornan más favorables.

En quinto lugar, sólo un indicador individual de acceso al 'capital cultural objetivado' libros+didacticos - reflejó alguna interacción con el contexto. La tenencia de libros y materiales escolares influye más intensamente en contextos académicos más elevados, y por el contrario, predice el rendimiento menos ajustadamente en los contextos académicos menos favorecidos. Sin embargo, dado la forma (subjetiva) de construcción de este indicador, la interpretación de este resultado requiere cierta cautela. La validez de uno de sus componentes - didácticos - puede estar afectada por la posible variación en la cantidad y la calidad objetivas que son solicitadas al alumno a medida que cambia el contexto.

Los rendimientos predichos por (educación padres) o por (bienes+servicios) se tornan también más homogéneos, pero en grados muy inferiores

Finalmente, los grados de (in)equidad sociocultural a nivel escuela serán más similares entre escuelas con igual 'composición' que en la muestra total de escuelas. En el caso particular del indicador libros+didácticos, la conclusión es aún más terminante: la intensidad de su efecto es la misma cuando se trata de escuelas con la misma 'composición'. Esta conclusión, sin embargo, no resultó confirmada para los otros factores de inequidad - historia académica, género y tiempo diario de trabajo extra-escolar.

Los datos analizados han permitido identificar diferentes aspectos de la (in)equidad en los resultados escolares: (i) Las probabilidades de aprendizaje en el nivel secundario están más fuertemente vinculadas a la institución educativa a la que se accede; (ii) la (in)equidad educativa opera principalmente a través de la segmentación sociocultural del entramado institucional; (iii) pero, al mismo tiempo, las escuelas difieren respecto del grado en que los factores individuales de inequidad inciden en la distribución de los aprendizajes y además, (iv) las escuelas se mostraron más eficaces con los alumnos de origen social favorecido, con los varones y con los no repitientes; ( $v$ ) la (in)equidad del género, la repitencia y el 'capital cultural objetivado' varían según ciertas características del contexto. 
Este conjunto de constataciones empíricas dan sustento a ciertos criterios metodológicos para orientar futuros estudios referidos a la equidad educativa. El más general es, sin dudas, la conveniencia de ir más allá del simple "control" socioeconómico individual y/o grupal. La idea de equidad educativa en el sistema educativo se despliega en una diversidad de aspectos, posibles de ser identificados y dimensionados a través de estudios "correlacionales". Haber encontrado variaciones en la equidad, no sólo a nivel escuela (aleatoriedad), sino también en relación a tipos de contextos (interacciones), aconseja profundizar el estudio de las posibles correlaciones entre los efectos de los factores individuales, y de estos con el rendimiento promedio de la escuela (análisis de covarianza a nivel escuela). Por otra parte, la detección de una mayor eficacia intra-escuela para los alumnos de origen social favorecido, para los varones y para los no repitientes sugiere la relevancia de profundizar el estudio de eficacia institucional por tipo de alumnos.

La variación de la inequidad institucional ha sido totalmente 'explicada' en un solo caso (libros y material didáctico); para el resto de los indicadores, la explicación ha sido mínima o inclusive, inexistente. Esto justifica futuros trabajos para explorar la posibilidad de que las diferencias en la capacidad igualitaria de las escuelas estén asociadas a factores escolares. Tal confirmación sería particularmente importante, pues constituiría un indicio de la magnitud del efecto que tendrían políticas y programas innovadores sobre los niveles de (in)equidad existente en el sistema. Al mismo tiempo, se justifican los estudios "cualitativos" (en profundidad) de aquellas 'escuelas eficaces' por su capacidad para promover la equidad, tendientes a proporcionar mayor comprensión de los procesos subyacentes a esas posibles asociaciones entre inequidad y factores escolares. Cualquiera sean los futuros hallazgos, las evidencias actuales sobre las diferencias institucionales en la capacidad distributiva exige que todo intento por evaluar a la escuela deba incluir como criterio el grado de inequidad.

Todas estas constataciones, sin embargo, se refieren exclusivamente a los resultados en la prueba de Matemática, y no pueden ser extrapolados sin más a otros resultados académicos o actitudinales de los alumnos. Quedan pendientes, pues, análisis que permitan extraer conclusiones más abarcadoras respecto a la inequidad en la educación Media de Argentina.

\section{BIBLIOGRAFÍA}

AITKING, M. y LONGFORD, N. (1986). Statistical modelling issues in school effectiveness. Journal of the Royal Statistical Society A, 149: 1-42.

BRYK, A. y RAUDENBUSH, S. (1992). Hierarchical Linear Models for Social and Behavioral Research: Applications and Data Analysis Methods. Newbury Park, CA:Sage.

CARROLL, J. (1963). A model of school learning, Teachers College Record, 43, pp.723-733.

CERVINI, R. (2001). Efecto de la 'Oportunidad de Aprender' sobre el Logro en Matemáticas de la Educación Básica de Argentina. Revista Electrónica de Investigación Educativa REDIE, 3(2): 1-22. México: Universidad Autónoma de Baja California. (http://redie.ens.uabc.mx)

CERVINI, R. (2002). Desigualdades Socioculturales en el Aprendizaje de Matemática y Lengua de la Educación Secundaria en Argentina: Un modelo de tres niveles. Revista Electrónica de Investigación y Evaluación Educativa, 8(2): 1-25. España: Universidad de Valencia 
CerVINI, R. (2003a.). Relaciones entre Composición escolar, Proceso escolar y el Logro en Matemática del nivel Secundario en Argentina - Un modelo de tres niveles. Revista Electrónica de Investigación Educativa, vol. 5(1). http://redie.ens.uabc.mx/vol5no1/contenido-cervini2.html

CERVINI, R. (2004) Influencia de los factores institucionales sobre el logro en Matemática de los estudiantes en el último año de la educación Media de Argentina - Un modelo de tres niveles. Revista Electrónica Iberoamericana sobre Calidad, Eficacia y Cambio en Educación. Vol. 2(1), pp. 1-24. España: Universidad de Deusto y Universidad de Madrid. http://www.ice.deusto.es/rinace/reice/vol2n1/Cervini.pdf

Cervini, R. y Basualdo, M. (2003b.) La eficacia educativa del sector público - El caso de las escuelas secundarias técnicas en Argentina". Revista Latinoamericana de Estudios Educativos, vol. XXXIII, № 3, pp. 53-92. México: Centro de Estudios Educativos.

COOPER, H., Valentine, J.C., NYE, B. y LINDSAY, J.J. (1999). Relationships between five after-school activities and academic achievement. Journal of Educational Psychology, 91(2), 369-378.

FREIDMAN, L. (1989). Mathematics and the gender gap: A meta-analysis of recent studies on sex differences in mathematical tasks. Review of Educational Research, 59, pp.185-213.

FENNEMA, E. y CARPENTER, T.P. (1981) Sex related differences in mathematics: Results from national assessment. Mathematics Teacher, 74: 554-559.

GERBER, S. (1996). Extracurricular activities and academic achievement. Journal of Research and Development in Education, 30(1), pp. 42-50.

GoldSTEIN, H. (1995). Multilevel Statistical Models. Londres: Edward Arnold.

Goldstein, H. Rasbash, J., Plawis, I., Draper, D., Browne, W., Yang, M., Woodhouse, G. y healy, M. (1998). A user Guide to MlwinN. Londres: University of London.

HILTON, T. L. y BERGLUND, G.W. (1974). Sex differences in mathematics achievement -e longitudinal study. The Journal of Educational Research, 67, pp. 232-237.

HOLLAND, A. y ANDRE, T. (1987). Participation in extracurricular activities in secondary school: What is known, what needs to be known? Review of Educational Research, 57, pp.437-466.

KABLAOUI, B.N. y PAUTLER, A.J. (1991). The effect of part-time work experience on high school students. Journal of Career Development, 17, pp.195-211.

LAM, R., WONG, K. y HO LAIM ING (2002) School effectiveness of a streamed-school system: A multilevel modelling of the Hong Kong secondary schools. Australian Journal of Education, 46(3), 287-304.

LEE, V. y BRYK, A. (1998). A multilevel model of the social distribution of high school achievement. Sociology of Education, Vol. 62 (July): 172-192.jError! Marcador no definido.

MARSH, H.W. (1992). Extracurricular activities: Beneficial extension of the traditional curriculum or subversion of academic goals? Journal of Educational Psychology; 84(4), pp. 553-562.

MARSH, H (1991) Employment during high school: Character building or a subversion of academic goals? Sociology of Education, 64: pp. 172-189. 
Mortimer, J.T., Finch, M.D., Ryu, S., Shanahan M.J. y Call, K.T. (1996). The effects of work intensity on adolescent mental health, achievement, and behavioral adjustment: New evidence from a prospective study. Child Development, 67: pp. 1243-1261.

Nuttall, D., Goldstein, H., Prosser, R. y RAsBash, J. (1989). Differential school effectiveness, International Journal of Educational Research. Vol.13 (7): 769-776.

OPDENAKKER, M. y VAN DAMME, J. (2001). Relationship between school composition and characteristics of school process and their effect on mathematics achievement. British Educational Research Journal, Vol. 27 (4): 407-432.

QUIRK, K.; KEITH, T. y QUIRK, J. (2002). Employment during high school and student achievement: Longitudinal analysis of national data. The Journal of Educational Research, 95 (1), pp. 4-10

SAMMONS, P., THOMAS, S. y MORTIMORE, P. (1997). Forging Link: Effective Schools and Effective Departments. Londres: Paul Chapman Publishing.

SINGH, K. (1998). Part-time employment in high school and its effect on academic achievement. The Journal of Educational Research, 91, 1 pp. 31-139.

SteinBERG, L. y KAUfMAN, E. (1995). The impact of employment on adolescent development. Annals of Child Development, 11, pp. 131-166.

STEINBERG, L. y DORNBUSH, S.M. (1991) Negative correlates of part-time employment during adolescence: Replication and elaboration. Development Psychology, 27, pp. 304-313.

SCHREIBER, JAMES B. (2002) Institutional and student factors and their influence on advanced mathematics achievement. The Journal of Educational Research, Vol. 95(5): 274-286.

THOMAS, S. (2001) Dimensions of Secondary School Effectiveness: Comparative Analyses across Regions, School Effectiveness and School Improvement, Vol. 12, № 3:285-322

TSAI, S.L. y WALBERG, H.J. (1983) Mathematic achievement and attitude productivity in junior high school. The Journal of Educational Research, 76, pp. 267-272.

VAN PARIJS, P. (1992). ¿Qué es una sociedad justa? Buenos Aires: Ediciones Nueva Visión.

\begin{tabular}{|c|c|c|c|c|c|c|}
\hline \multicolumn{7}{|c|}{ Anexo A - Coeficientes de correlación de variables del alumnos y de la escuela } \\
\hline Variables & femenino & repitiente & $\begin{array}{l}\text { libros+ } \\
\text { didacticos }\end{array}$ & hs_trabajo & $\begin{array}{l}\text { bienes+ } \\
\text { servicios }\end{array}$ & $\begin{array}{l}\text { educació } \\
\text { n padres }\end{array}$ \\
\hline Femenino & 1 & ---- & ---- & ----- & ----- & ---- \\
\hline Repitiente & -0.114 & 1 & ----- & ----- & ----- & ---- \\
\hline libros+didacticos & 0.091 & -0.458 & 1 & -0.112 & 0.250 & 0.2655 \\
\hline hs_trabajo & -0.106 & 0.650 & -0.516 & 1 & -0.148 & -0.194 \\
\hline bienes+servicios & -0.125 & -0.540 & 0.737 & -0.633 & 1 & 0.507 \\
\hline educacion padres & -0.122 & -0.556 & 0.680 & -0.665 & 0.898 & 1 \\
\hline
\end{tabular}




\begin{tabular}{|c|c|c|c|c|c|c|c|c|c|c|c|c|}
\hline \multirow{3}{*}{\multicolumn{2}{|c|}{\begin{tabular}{|c|} 
Anexo B - Coeficientes y \\
Indicadores y niveles
\end{tabular}}} & error $\epsilon$ & stánda & rde $l$ & mode & $\operatorname{los} m$ & ultinivel & , seg & ún obje & etivos & del es & tudio. \\
\hline & & 1 & \multicolumn{2}{|c|}{2} & \multicolumn{2}{|c|}{3} & \multicolumn{2}{|l|}{4} & \multicolumn{2}{|c|}{5} & \multicolumn{2}{|c|}{6} \\
\hline & & Coef. & Coef. & e.s. & Coef. & e.s. & Coef. & e.s. & Coef. & e.s. & Coef. & e.s. \\
\hline \multicolumn{2}{|c|}{ PARTE FIJA (Prom.) } & 58,30 & 52,05 & $\begin{array}{l}1,48 \\
2\end{array}$ & & & 30,60 & 3.36 & 33,41 & $\begin{array}{l}3,90 \\
4 \\
\end{array}$ & & \\
\hline \multicolumn{2}{|l|}{ INDIVIDUAL } & & & & & & & & & & & \\
\hline \multicolumn{2}{|c|}{ 1. educación padres } & & 0.533 & .018 & & & 0.498 & .018 & 0.489 & .018 & & \\
\hline \multicolumn{2}{|c|}{ 2. bienes+servicios } & & 0.119 & .021 & & & 0.087 & .021 & 0.082 & .021 & & \\
\hline \multicolumn{2}{|c|}{ 3. $h s$ trabajo } & & -0.477 & .031 & & & -0.442 & .031 & -0.444 & .031 & & \\
\hline \multicolumn{2}{|c|}{ 4. libros +didacticos } & & 1.424 & .068 & & & 1.384 & .068 & 0.907 & .845 & & \\
\hline \multicolumn{2}{|l|}{ 5. repitiente } & & -4.845 & .109 & & & -4.664 & .109 & -2.867 & .715 & & \\
\hline \multicolumn{2}{|l|}{ 6. femenino } & & -1.226 & .095 & & & -1.231 & .095 & -4.251 & .739 & & \\
\hline \multicolumn{2}{|l|}{ COMPOSICIÓN } & & & & & & & & & & & \\
\hline \multicolumn{2}{|c|}{ 1. educación padres } & & & & & & 0.971 & .259 & 0.802 & .173 & & \\
\hline \multicolumn{2}{|c|}{ 2. bienes+servicios } & & & & & & -0.178 & .327 & & & & \\
\hline \multicolumn{2}{|c|}{ 3. $h s$ trabajo } & & & & & & -2.068 & .512 & -2.650 & .527 & & \\
\hline \multirow{2}{*}{\multicolumn{2}{|c|}{$\begin{array}{l}\text { 4. libros+didacticos } \\
\text { 5. repitiente }\end{array}$}} & & & & & & 8.539 & 1.24 & 6.923 & 1.43 & & \\
\hline & & & & & & & -12.81 & 1.31 & -9.316 & 1.71 & & \\
\hline \multicolumn{2}{|l|}{ 6. femenino } & & & & & & -1.739 & 1.06 & & & & \\
\hline \multicolumn{2}{|l|}{ INTERACCION } & & & & & & & & & & & \\
\hline femexeduc_pror & & & & & & & & & 0.349 & 0.07 & & \\
\hline femexhs_trab_p & & & & & & & & & 1.024 & 0.27 & & \\
\hline femex\%repi & & & & & & & & & -2.802 & 0.67 & & \\
\hline repix\%repi & & & & & & & & & 4.363 & 0.71 & & \\
\hline Repixeduc_prom & & & & & & & & & -0.453 & 0.08 & & \\
\hline librox\%repi & & & & & & & & & -1.125 & 0.39 & & \\
\hline libroxlibro_prom & & & & & & & & & 0.278 & 0.29 & & \\
\hline PARTE ALEATO & & & & & & & & & & & & \\
\hline Estado (\%) & & 46.52 & 39.44 & 13.4 & & & 22.981 & 7.98 & 23.39 & 8.08 & & \\
\hline Escuela (\%) & & 126.4 & 97.52 & 3.15 & & & 66.262 & 2.19 & 65.94 & 2.18 & & \\
\hline educación & Intercepto & & & & 93.777 & $\begin{array}{c}3.35 \\
6\end{array}$ & & & & & 63.11 & 2.386 \\
\hline de padres & Pendiente & & & & 137 & $\begin{array}{c}0.01 \\
8\end{array}$ & & & & & 0.107 & 0.016 \\
\hline Bienes+ & Intercepto & & & & 92.427 & $\begin{array}{c}3.33 \\
8\end{array}$ & & & & & 61.64 & 2.435 \\
\hline servicios & Pendiente & & & & 0.110 & $\begin{array}{c}0.01 \\
9\end{array}$ & & & & & 0.089 & 0.017 \\
\hline hs trabaio & Intercepto & & & & 97.146 & $\begin{array}{c}3.15 \\
8\end{array}$ & & & & & 64.95 & 2.160 \\
\hline 15_travajo & Pendiente & & & & 0.674 & $\begin{array}{c}0.07 \\
7\end{array}$ & & & & & 0.698 & 0.077 \\
\hline Libros+ & Intercepto & & & & 92.548 & $\begin{array}{c}3.43 \\
7\end{array}$ & & & & & 64.14 & 2.482 \\
\hline didácticos & Pendiente & & & & .930 & .201 & & & & & 0.340 & 0.162 \\
\hline renitic & Intercepto & & & & 97.815 & $\begin{array}{c}3.18 \\
2\end{array}$ & & & & & 64.47 & 2.156 \\
\hline repritane & Pendiente & & & & 12.677 & $\begin{array}{c}1.14 \\
2\end{array}$ & & & & & 12.61 & 1.132 \\
\hline & Intercepto & & & & 94.172 & $\begin{array}{c}3.15 \\
8 \\
\end{array}$ & & & & & 63.95 & 2.217 \\
\hline temenino & Pendiente & & & & 12.142 & $\begin{array}{c}0.91 \\
8\end{array}$ & & & & & 10.46 & 0.840 \\
\hline Alumno (\%) & & 226.0 & 218.5 & 0.88 & & & 218.43 & 0.88 & 218.1 & 0.87 & & \\
\hline Tests verosimili & & $\begin{array}{c}105268 \\
4\end{array}$ & 10479 & 03.0 & & & 10471 & 15.0 & 1046 & 902 & & \\
\hline
\end{tabular}




\section{PUBLICACIONES RECIENTES SOBRE 'FACTORES DEL RENDIMIENTO’}

I. Capítulos de libro:

(2002) "La distribución social de los rendimientos escolares". En: El rendimiento escolar en Argentina Análisis de resultados y factores. Emilio Tenti (Org.), Cap.IV: 177-230. Buenos Aires: Editorial Losada.

(2002) "Los factores del rendimiento en la educación básica de Argentina." En: El rendimiento escolar en Argentina - Análisis de resultados y factores. Emilio Tenti (Org.), Cap. V: 231-281. Buenos Aires: Editorial Losada.

\section{Artículos publicados}

(2001) "Efecto de la 'Oportunidad de Aprender' sobre el Logro en Matemáticas de la Educación Básica de Argentina." Revista Electrónica de Investigación Educativa REDIE, 3 (2): 1-22. México: Universidad Autónoma de Baja California.

(http://redie.ens.uabc.mx)

(2002) "Desigualdades Socioculturales en el Aprendizaje de Matemática y Lengua de la Educación Secundaria en Argentina: Un modelo de tres niveles." Revista Electrónica de Investigación y Evaluación Educativa, 8(2):1-25. España: Universidad de Valencia. http://www.uv.es/RELIEVE/v8n1/RELIEVEv8n2 1. htm

(2002) "Desigualdades en el Logro Académico y Reproducción Cultural en la Educación Primaria de Argentina - Un modelo de tres niveles." Revista Mexicana de Investigación Educativa, 7(16): 445-500 México: Consejo Mexicano de Investigación Educativa. http://www.comie.org.mx/revista/Pdfs/Carpeta16/16investTem2.pdf

(2002) "Participación familiar y logro académico del alumno". Revista Colombiana de Educación, № 43: 93133. ISSN 0120-3916. Colombia: Universidad Pedagógica Nacional.

(2003) "Diferencias de resultados cognitivos y no-cognitivos entre estudiantes de escuelas públicas y privadas en la educación secundaria de Argentina: Un análisis multinivel." Education Policy Analysis Archives, 11(5).

http://epaa.asu.edu/epaa/v11n6/.

(2003) "Relaciones entre Composición escolar, Proceso escolar y el Logro en Matemática del nivel Secundario en Argentina - Un modelo de tres niveles" Revista Electrónica de Investigación Educativa, vol.5(1)

http://redie.ens.uabc.mx/vol5no1/contenido-cervini2.html

(2003) La eficacia educativa del sector público - El caso de las escuelas secundarias técnicas en Argentina". Revista Latinoamericana de Estudios Educativos, vol. XXXIII, № 3, pp. 53-92. México: Centro de Estudios Educativos.

(2004) Influencia de los factores institucionales sobre el logro en Matemática de los estudiantes en el último año de la educación Media de Argentina - Un modelo de tres niveles. Revista Electrónica Iberoamericana sobre Calidad, Eficacia y Cambio en Educación. Vol. 2(1), pp. 1-24. España: 
Universidad de Deusto y Universidad de Madrid.

http://www.ice.deusto.es/rinace/reice/vol2n1/Cervini.pdf

(2004) Factores Asociados al Aprendizaje del Lenguaje y las Matemáticas en 13 Estados de México (3ํy 4ํㅡㅁodos en Educación Básica). Colección Cuadernos de Investigación, no 7, pp.1-22. México: Instituto Nacional para la Evaluación de la Educación (INEE).

http://www.capacitacion.ilce.edu.mx/inee/cuadrno/cuaderno 7.pdf 


\title{
Contactar
}

Revista lberoamericana de Educación

\author{
Principal OEI
}

